\title{
PD-L1 biology in response to chemotherapy in vitro and in vivo in ovarian cancer
}

\author{
Shannon Grabosch ${ }^{*}$, Feitianzhi Zeng ${ }^{2}$, Lixin Zhang ${ }^{2}$, Mary Strange ${ }^{2}$, Joan Brozick², Robert P Edwards ${ }^{3}$, Anda Vlad ${ }^{2}$ \\ From 30th Annual Meeting and Associated Programs of the Society for Immunotherapy of Cancer (SITC 2015) \\ National Harbor, MD, USA. 4-8 November 2015
}

\section{Objective}

PD-L1 is an immune checkpoint molecule expressed by a variety of tumors, including ovarian, which binds to circulating PD-1 expressing effector T cells allowing for tumor escape from the immune system. PD-L1 blockade prevents $\mathrm{PD}-\mathrm{L} 1 / \mathrm{PD}-1$ interaction and is currently explored as therapy of solid tumors. Ovarian cancer patients receive combination cisplatin/taxane chemotherapy as standard of care. Chemo-induced effects on tumor PD-L1 expression have been only partially addressed. We studied here the effect of platinum/taxane exposure on PD-L1 expression in vitro and in vivo.

\section{Methods}

Human (OVCA 420 and OVCA432) and mouse (2F8) ovarian cancer cell lines were exposed to increasing doses of cisplatin and paclitaxel for different time periods. PD-L1 expression was analyzed with flow cytometry and Western blot. Through continuous exposure in vitro of mouse 2F8 ovarian cancer cells to increasing doses of cisplatin we have derived a new cisplatin-resistant line (2F8Cis). In vivo, we have challenged $n=37$ mice IP with 0.8 million 2F8 cells. Tumor-bearing mice were treated with cisplatin, anti-PD-L1 antibody, both drugs, or isotype control every two weeks for three doses starting at day 14 post-inoculation. Tumor- and ascites-derived cancer cells were analyzed with flow cytometry.

\section{Result}

Exposure of OVCA420 and OVCA432 to cytotoxic doses of cisplatin or paclitaxel trigger PD-L1 up-regulation. Similarly, 2F8-Cis cells show increased cell surface PD-L1 compared to parental $2 \mathrm{~F} 8$ cells, providing the rationale for combination therapy with PD-L1 blockade. In vivo treatment of mice with aggressive $2 \mathrm{~F} 8$ tumors respond well to cisplatin and anti-PD-L1 individually with increased survival (median 45 days versus 24 days for isotype control, $\mathrm{p}=0011$ ). At necropsy, anti-PD-L1 therapy significantly reduced tumor burden $(1.48 \mathrm{~g}$ versus $0.25 \mathrm{~g}$, $\mathrm{p}=0.0294$ ). Tumor cells cultured from cisplatin-only treated mice expressed higher levels of PD-L1, in line with our in vitro results. A higher percentage of PD-1 expressing cells were found amongst the tumor cells in these cultures versus cisplatin/anti-PD-L1 treated mice. Although high dose anti-PD-L1 immediately following cisplatin administration can control tumor burden $(0.48 \mathrm{~g})$, it does not significantly prolong survival (median 29 days). We are currently testing an alternative therapeutic schema exploring a lower anti-PD-L1 dose and a different timing post-chemo.

\section{Conclusion}

Tumor cells upregulate PD-L1 in response to chemotherapy exposure and combination PD-L1 blockade in conjunction with chemotherapy effectively controls tumor burden. Optimization of timing and dosage for this combination therapy will likely increase its therapeutic benefit.

\section{Authors' details}

${ }^{1}$ Magee-Womens Hospital of UPMC, Pittsburgh, PA, USA. ${ }^{2}$ Magee-Womens Research Institute, Pittsburgh, PA, USA. ${ }^{3}$ Magee-Womens Research Institute Ovarian Cancer Center of Excellence, Pittsburgh, PA, USA.

Published: 4 November 2015

doi:10.1186/2051-1426-3-S2-P302

Cite this article as: Grabosch et al:: PD-L1 biology in response to

chemotherapy in vitro and in vivo in ovarian cancer. Journal for

ImmunoTherapy of Cancer 2015 3(Suppl 2):P302.

${ }^{1}$ Magee-Womens Hospital of UPMC, Pittsburgh, PA, USA

Full list of author information is available at the end of the article 